\title{
Formation et propriétés de l'interface métal-semiconducteur et de la barrière de Schottky
}

\author{
L. Lassabatère \\ Laboratoire d'études des surfaces, interfaces et composants - unité associée CNRS 04-0787, Université des \\ Sciences et des Techniques du Languedoc, place Eugène-Bataillon, 34060 Montpellier, France
}

(Reçu le 2 février 1987, révisé le $1^{\text {er }}$ avril 1987, accepté le 30 juin 1987)

\begin{abstract}
Résumé. - Après un rappel succinct de l'histoire du contact destiné à mettre en évidence la façon dont s'est effectuée la prise de conscience sur le rôle des différents paramètres et la démarche de recherche qui en a résulté, on s'attache tout d'abord, à l'aide de quelques exemples, à illustrer la méthodologie actuelle basée sur un suivi pas à pas de la formation du contact. On présente donc succinctement des méthodes d'études de propriétés de surfaces, des toutes premières phases de l'interaction, de la formation de la diode, ainsi que quelques résultats significatifs. On s'attache ensuite à l'analyse des résultats et expose les différents modèles, anciens comme tout récents, utilisés pour les expliquer. On conclut qu'aucun modèle à l'heure actuelle ne permet une synthèse correcte de l'ensemble des résultats, ce qui implique un effort de recherche accru.
\end{abstract}

\begin{abstract}
In this paper we review fondamental points in the formation of Metal Semiconductor contact. After a brief historical view in order to emphasize the role of physical and chemical parameters we describe typical results concerning the formation, stage by stage, of the III-V metal contacts. We compare the surface Fermi level pinning induced by cleavage defects, oxygen adsorption, metal submonolayer deposition and the pinning in the diodes. Then we briefly present and discuss the results and the models which are used to explain them. We conclude that, at this time, there is no synthetical model able to explain all the experimental results.
\end{abstract}

Dans la physique moderne, comme dans l'électronique des composants, les interfaces ont une part dont l'importance a augmenté de façon exceptionnelle dans la dernière décennie. Ceci résulte d'une part, d'une demande venant de l'aval, au niveau de la compréhension des mécanismes en vue d'une meilleure maîtrise de la technologie et de l'amélioration des performances des composants, d'autre part, d'une avancée des études fondamentales vers des systèmes de dimensionalité réduite, avancée permise ou induite par la meilleure connaissance des propriétés de volume et aussi par l'évolution des techniques d'élaboration.

Parmi ces interfaces certaines comme le contact métal-semiconducteur ou comme l'interface silicium-silice étaient déjà au centre des préoccupations de nombreux groupes depuis de nombreuses années, les années 60 , ou même avant. D'autres, comme celles que l'on trouve dans les structures à interfaces multiples, comme les super-réseaux par exemple, ne donnent lieu à des travaux denses que depuis quelques années seulement.

A l'heure actuelle, et malgré l'antériorité des REVUE DE PHYSIQUE APPLIQUÉE. - T. 22, N` 11, NOVEMBRE 1987 études sur le contact métal-semiconducteur, les travaux sur ce sujet sont tout aussi nécessaires que ceux consacrés aux structures plus nouvelles. En effet, en raison de la complexité des mécanismes et de la multiplicité des paramètres, la connaissance de ce contact est très loin d'être complète. Et pourtant, ce contact est le plus élémentaire. Il peut mettre en jeu un nombre très limité d'atomes de nature différente ( $\mathrm{Si}+\mathrm{Métal}=2$ types d'atomes). Il permet de réaliser des combinaisons multiples en élaborant des surfaces semiconductrices de propriétés variées, en prenant en compte la structure électronique des atomes, leur réactivité, leurs dimensions... et de préciser ainsi sélectivement le rôle de quelques-uns des différents paramètres qui jouent sur les propriétés de l'interface.

C'est donc un thème porteur en raison de sa structure simple et de son intérêt au plan des composants. Ceci explique les études multiples qui lui ont été consacrées, depuis de nombreuses années, et le regain d'intérêt dont il est l'objet ces derniers temps. Ce regain d'intérêt s'est traduit par l'apparition d'analyses nouvelles, par la proposition de 
modèles conçus à partir de résultats expérimentaux obtenus par une étude pas à pas in situ de l'interface en cours de formation, par une analyse de l'évolution de la structure électronique des atomes de surface, des potentiels de surface, de la physicochimie, de la croissance et de la morphologie de la couche métallique, enfin par une étude plus fouillée de la diode finie.

Ces recherches dont des bilans synthétiques sont présentés dans d'excellents livres tout récemment publiés [1,2] ont pu se développer grâce à une avancée considérable des techniques d'analyse et des méthodes d'élaboration par jets moléculaires en particulier. Elles ont permis d'obtenir des résultats substantiels. Malgré ce, au plan de la physique comme au plan de la modélisation, il n'existe pas, à ce jour, de réponses claires aux problèmes posés. Le contact métal-semiconducteur est au centre d'un grand débat dont cet article s'efforcera de dégager les éléments essentiels. Il comportera pour cela après un bref rappel sur les paramètres jouant sur le contact et sur son histoire, une présentation de la formation de l'interface illustrée par quelques exemples et une description des modèles utilisés pour en expliquer les propriétés.

\section{Les paramètres jouant sur le contact.}

Lorsqu'un atome métallique tombe sur une surface semiconductrice, il peut, si le coefficient de collage n'est pas égal à 1 , être réfléchi ou se fixer sur la surface. Dans ce dernier cas, la liaison qui le retient peut être faible (quelques centièmes d'eV par exemple) dans le cas de physisorption, un peu plus forte dans le cas où il y a échange de charge entre la population de porteurs libres et l'atome, plus élevée lorsqu'il y a formation de liaisons localisées sur des sites de surface. La nature de l'adsorption sera liée aux propriétés volumiques du semiconducteur, à la nature des sites de surfaces, aux propriétés de l'atome métallique et à des paramètres macroscopiques comme, par exemple, la température. La composition de la surface, sa structure, ses défauts, son état électronique et ses liaisons pendantes pourront contribuer de façon spécifique à la première phase de l'interaction, celle qui correspond à un faible recouvrement.

Au cours de l'étape suivante, plusieurs possibilités sont à envisager. L'atome adsorbé peut, et cela dépend de la force qui le lie au substrat, du recouvrement de la surface et donc des forces d'interaction entre atomes métalliques, de la température, rester sur son site d'adsorption, diffuser le long de la surface pour former des amas, diffuser vers l'intérieur du semiconducteur en se plaçant en position interstitielle. Il peut également si la force de liaison interatomique du semiconducteur n'est pas trop importante, contribuer à la rupture de ses liaisons et former un composé nouveau, cette formation pouvant s'initier par le premier plan de surface ou par des plans sous-jacents. Interviennent donc, dans ce processus, la réactivité du métal, sa chaleur de condensation, la chaleur de formation du composé nouveau comparée à celle du semiconducteur, bref la réactivité de l'interface étalonnée à partir de la différence entre ces deux quantités.

Globalement donc, suivant les matériaux en présence, suivant les conditions expérimentales, on pourra avoir affaire à des interfaces abruptes, le passage du métal vers le semiconducteur se faisant sur un tout petit nombre de plans atomiques ou à des interfaces graduelles, voire à des interfaces tout à fait spécifiques dans le cas où un composé nouveau en surface isole l'électrode métallique du semiconducteur.

Ces quelques remarques tout à fait générales amènent naturellemẹt à envisager une corrélation entre les propriétés de la diode finie, sa barrière $\phi_{\mathrm{B}}$ et la nature des éléments en présence. C'est ce qui a été effectivement fait au cours du temps. Il en résulte quelques analyses qui ont ensuite servi de base à des appréciations plus récentes. Nous en résumerons donc, de façon succincte les grandes lignes.

\section{Historique.}

Les premiers éléments de corrélation ont été établis par Schottky [3], Mott [4], qui ont cherché à établir une relation entre la hauteur de barrière et la différence entre le travail de sortie du métal $\phi_{M}$ et celui du semiconducteur $\phi_{\text {SC }}$. Effectivement, dans le cas de certains contacts, il se forme une barrière de diffusion dans le semiconducteur qui s'ajuste de façon à rendre son travail de sortie après contact $\phi_{\mathrm{SC}}$ égal à celui du métal.

On a alors, avec les notations précises (Fig. 1)

$$
\begin{gathered}
\phi_{\mathrm{M}}=X-q V_{\mathrm{s}}+\left(E_{\mathrm{C}}-E_{\mathrm{FSC}}\right)_{0}=\phi_{\mathrm{SC}} \\
\phi_{\mathrm{B}}=\phi_{\mathrm{M}}-X=S\left(\phi_{\mathrm{M}}-\phi_{\mathrm{SC} 0}\right)+C
\end{gathered}
$$

avec

$$
\left(E_{\mathrm{C}}-E_{\mathrm{FSC}}\right)_{0}=C
$$

et $S$ indice de comportement de l'interface $=$ $\frac{\mathrm{d} \phi_{\mathrm{B}}}{\mathrm{d} \phi_{\mathrm{M}}}=1$.

En fait cette corrélation n'est valable que dans quelques cas et en particulier, lorsque le substrat est un matériau à forte liaison sur lequel le métal se lie très faiblement, voire se physisorbe $\left(\mathrm{Au} / \mathrm{SiO}_{2}\right.$, $\left.\mathrm{Al} / \mathrm{SiO}_{2} \ldots\right)$.

De façon plus générale, $S$ est différent de 1 . On ne retrouve pas dans la barrière les variations du travail de sortie du métal. Ceci a conduit Mead [5], à introduire l'électronégativité du métal $X_{\mathrm{M}}$, connue de façon plus certaine que $\phi_{M}$, puis [6] la différence 

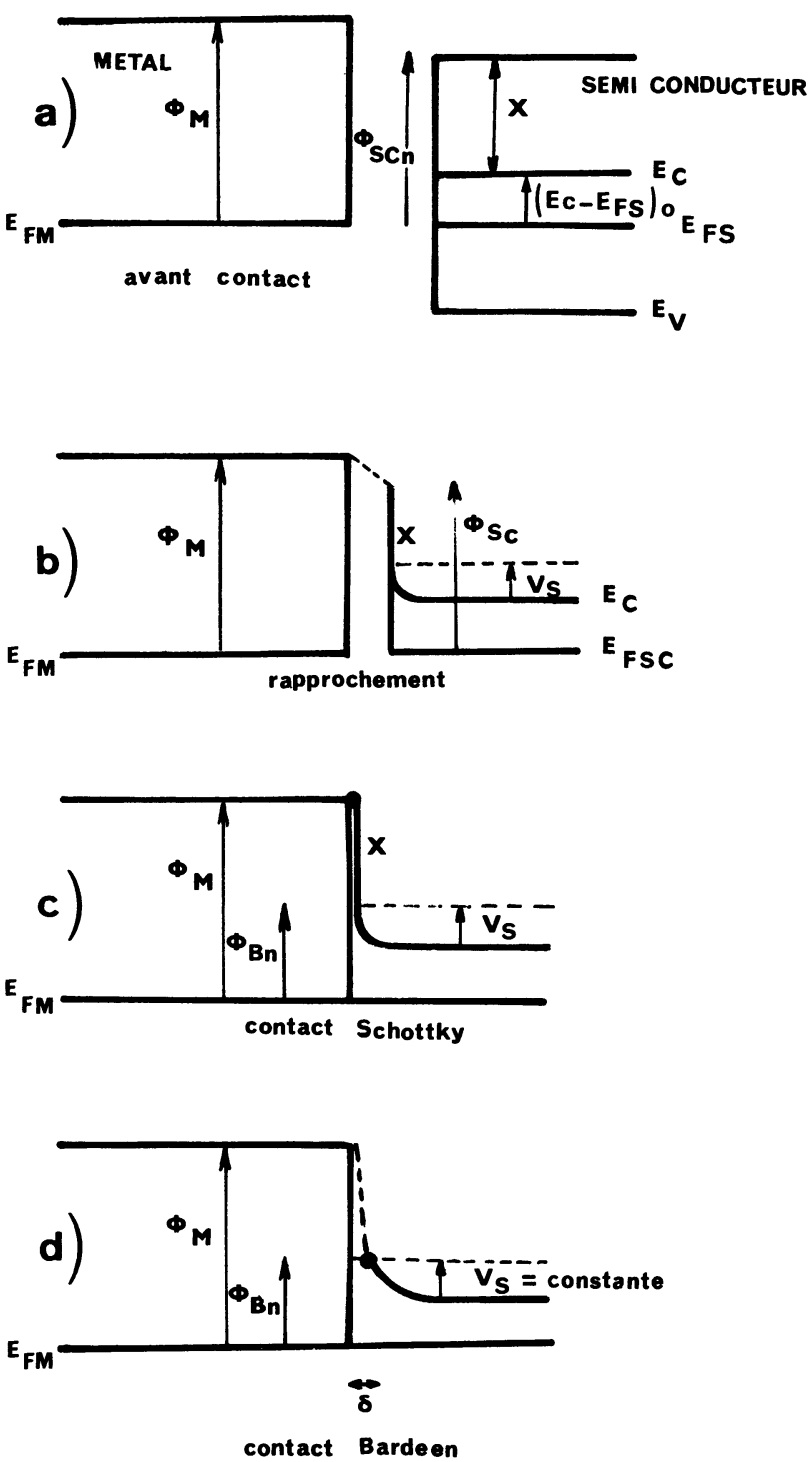

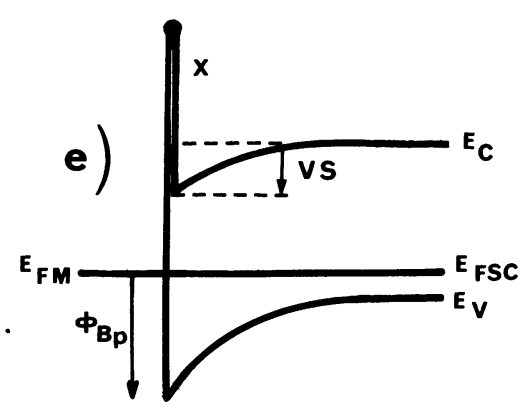

Fig. 1. - Contact metal semiconducteur

(a) Diagramme de bande : métal et semiconducteur de type $n$ éloignés.

(b) Diagramme de bande : métal et semiconducteur de type $\mathrm{n}$ très rapprochés.

(c) Diagramme de bande : contact Schottky sur semiconducteur de type $\mathrm{n}$.

(d) Diagramme de bande : contact Bardeen sur semiconducteur de type $n$.

(e) Diagramme de bande : contact Schottky sur semiconducteur de type $p$.

$\phi_{\mathrm{Bn}} \phi_{\mathrm{Bp}}$ Barrière de Schottky respectivement sur semiconducteur de type $n$ et $p$.

[Metal semiconductor contact

(a) Energy band diagram before contact.

(b) Energy band diagram when decreasing the distance semiconductor metal.

(c) Energy band diagram : Schottky contact on $n$ type semiconductor.

(d) Energy band diagram : Bardeen contact on $n$ type semiconductor.

(e) Energy band diagram : Schottky contact on $p$ type semiconductor.

$\phi_{\mathrm{Bn}} \phi_{\mathrm{Bp}}$ Schottky barriers on $\mathrm{n}$ and $\mathrm{p}$ type semiconductor.] entre les électronégativités $X_{\mathrm{A}}$ et $X_{\mathrm{B}}$ des atomes, AB par exemple, constituant le semiconducteur. $S^{*}=\frac{\mathrm{d} \phi_{\mathrm{B}}}{\mathrm{d} X_{\mathrm{m}}}$ s'avère alors égal à 1 dans le cas des matériaux ioniques pour lesquels $\left|X_{\mathrm{B}}-X_{\mathrm{A}}\right|>0,7$ (cas de $-\mathrm{SiO}_{2} \mathrm{SnO}_{2}, \mathrm{Al}_{2} \mathrm{O}_{3} \ldots$ ), très peu différent de zéro pour les matériaux covalents ( $\mathrm{Si}, \mathrm{Ge}, \mathrm{GaAs}$, ...) caractérisés par $\left|X_{\mathrm{B}}-X_{\mathrm{A}}\right|<0,7$ et dont la barrière est ancrée par une forte densité d'états d'interface. La limite correspondante est la limite de Bardeen [7].

Dans les cas intermédiaires $\phi_{\mathrm{B}}$ s'exprimera sous la forme

$$
\phi_{\mathrm{B}}=S^{*} X_{\mathrm{M}}+\phi_{0}
$$

$\phi_{0}$ traduisant alors la contribution des états d'interface.

Les deux cas limites ci-dessus mettent en évidence la contribution de la force de liaison entre les atomes du semiconducteur. Le fait que $\phi_{0}$ soit très dépendant de $X_{\mathrm{M}}$ pour les matériaux dont la liaison est fortement ionique, et indépendant pour les matériaux covalents conduit à préciser le rôle de la force de liaison. Dans cette optique, en 1975, Phillips et al. [8], après avoir établi une relation entre $S$ et la polarisabilité du semiconducteur $(1-S$ croît avec la constante diélectrique du semiconducteur) ont dans le cas de $\mathrm{Si}$ et des métaux de transition mis en évidence une corrélation entre $\phi_{\mathrm{B}}$ et la chaleur de formation $H_{\mathrm{F}}$ du siliciure, $\phi_{\mathrm{B}}$ devenant d'autant plus faible que $H_{\mathrm{F}}$ est négative. Les interfaces en question sont réactives et caractérisées par la formation d'une couche interfaciale qui fait que l'interface vraie se trouve repoussée à l'intérieur du semiconducteur et, de ce fait, indépendante des propriétés de la surface et du travail de sortie du métal.

Cette appréhension a été reprise par Brillson en 1978 [9] qui a étendu cette analyse aux alliages III-V et II-VI et établi une série de courbes en forme de S qui montrent que $\phi_{\mathrm{B}}$ croît respectivement avec la chaleur de formation du semiconducteur et la chaleur de réaction d'interface $\Delta H_{\mathrm{r}}$ définies par les relations 


$$
\begin{gathered}
M+\frac{1}{x} C A \rightarrow \frac{1}{x}\left[M_{\mathrm{x}} A+C\right] \\
\Delta H_{\mathrm{r}}=\frac{1}{x}\left[H_{\mathrm{F}}(C A)-H_{\mathrm{F}}\left(M_{\mathrm{x}} A\right)\right] .
\end{gathered}
$$

La barrière maximum lorsque les interfaces ne sont pas réactives, diminue fortement lorsque la réactivité croît. Parallèlement, la largeur de l'interface décroît avec la chaleur de réaction, les interfaces les plus abruptes étant caractéristiques des systèmes pour lesquels l'interaction métal-anion est la plus forte.

Les analyses précédentes supposent une croissance métallique couche par couche, sans îlots, ce qui ne correspond pas à l'ensemble des cas expérimentaux. De plus, les données thermodynamiques qu'elles utilisent correspondent à des réactions de volume. Malgré ce, elles mettent en évidence l'impact des interactions interfaciales sur la barrière et elles ont donné lieu à des développements nouveaux [10, 11]. Il faut enfin noter que des réévaluations récentes [12, 13 ] ont envisagé des cas pour lesquels $S$ pourrait être supérieur à 1 . La question de la barrière est donc toujours cruellement posée.

Les approches ci-dessus ont été développées dans l'hypothèse d'un premier contact métal-semiconducteur direct. Il n'en est pas ainsi lorsque la surface du semiconducteur est recouverte de sa couche d'oxyde natif, par exemple, ou d'une couche isolante mince d'une autre origine. Ce cas très important du point de vue expérimental a été traité par Cowley et Sze [14] qui ont proposé un modèle bien adapté [15] qui, aux limites, rend compte aussi des interactions correspondant aux modèles de Schottky et de Bardeen.

\section{De la surface à l'interface finie.}

L'approche précédente, très macroscopique, s'est très rapidement avérée limitée. On a alors été conduit à étudier, pas à pas, des interfaces en formation en cherchant à mettre en évidence la contribution des différents paramètres précédemment introduits. Pour cela, on part donc de la surface, on la caractérise. On effectue ensuite les dépôts à faible vitesse et précise les mécanismes de croissance du métal et les modifications de propriétés qui y sont associées. On construit la diode, la caractérise et cherche une corrélation éventuelle entre les différents résultats obtenus. Nous allons décrire les caractéristiques fondamentales de cette démarche et quelques-uns des résultats qu'elle fournit en nous limitant cependant aux contacts métalmatériaux III-V.

\subsection{SURFACE LIBRE.}

3.1.1 Caractéristiques essentielles. - Les études les plus complètes ont été effectuées sur des surfaces clivées (110) qui sont constituées d'un nombre égal d'atomes de la colonne III (cations : C) et d'atomes de la colonne V (anions : A) alors que les surfaces polaires (001) et (111) sont composées de couches successives complètes d'atomes III ou V.

Dans le cas général, la surface 110 n'a pas sa structure idéale, mais une structure relaxée [16]. La surface est reconstruite ; les atomes de la colonne $\mathrm{V}$ sont déplacés vers l'extérieur, ceux de la colonne III vers l'intérieur, ce qui se traduit par l'apparition d'un dipôle (dont la longueur peut atteindre 0,6 à $0,7 \AA$ ). Il en résulte une différence de potentiel supplémentaire à la traversée de la surface qui peut atteindre plusieurs dizaines, voire plusieurs centaines de meV. Cette relaxation a de plus un effet primordial sur les états électroniques intrinsèques de surface. Des calculs théoriques, confirmés par des résultats expérimentaux ont, en effet, montré que les états électroniques de surface de $\mathrm{GaAs}$, InP, InAs, InSb étaient localisés dans la bande de conduction et de valence. GaP, seule exception, présente une bande vide d'états dans le gap [17].

Cette absence d'états dans la bande interdite est fortement mise en évidence par des mesures de travail de sortie. En effet, en l'absence d'états, il ne doit pas $y$ avoir de barrière de surface et le travail de sortie est alors

$$
\begin{aligned}
\phi_{\mathrm{SC}}=X-q V_{\mathrm{s}}+\left(E_{\mathrm{C}}-E_{\mathrm{FSC}}\right)_{0}=X+ \\
+\left(E_{\mathrm{C}}-E_{\mathrm{FSC}}\right)_{0}=\phi_{\mathrm{SC} 0} .
\end{aligned}
$$

$\mathrm{Si}$ on compare les travaux de sortie $\phi_{\mathrm{SC} 1}$ et $\phi_{\mathrm{SC} 2}$ de deux échantillons de dopage différent, on ạra alors

$$
\phi_{\mathrm{SC} 1}-\phi_{\mathrm{SC} 2}=\left(E_{\mathrm{C}}-E_{\mathrm{FSC}}\right)_{01}-\left(E_{\mathrm{C}}-E_{\mathrm{FSC}}\right)_{02} .
$$

En fait des échantillons de type $n$ ne pouvant révéler que les accepteurs et les échantillons de type $\mathrm{p}$, que des donneurs (dans la mesure encore où ils ne sont pas respectivement très haut ou très bas dans le gap), la comparaison pour être parfaitement concluante doit être faite entre les échantillons de type $\mathrm{p}$ et de type $\mathrm{n}$.

La figure 2 représente des topographies du travail de sortie relevées par la méthode de Kelvin, en déplaçant une sonde devant la surface. Elle fait apparaître des zones de surface caractérisées par :

$$
\phi_{\mathrm{SCp}}-\phi_{\mathrm{SCn}}=\left(E_{\mathrm{C}}-E_{\mathrm{Fp}}\right)_{0}-\left(E_{\mathrm{C}}-E_{\mathrm{Fn}}\right)_{0}=\Delta \phi_{0}
$$

et d'autres par :

$$
\Delta \phi=\phi_{\mathrm{p}}-\phi_{\mathrm{n}}<\Delta \phi_{0}
$$

$\Delta \phi$ pouvant même devenir très peu différent de 0 . Ces variations de $\Delta \phi$ que l'on corrèle aisément à la qualité cristallographique locale de la surface, montrent que dans les zones parfaitement clivées, il n'y a pas d'états dans le gap, alors que dans les zones mal 

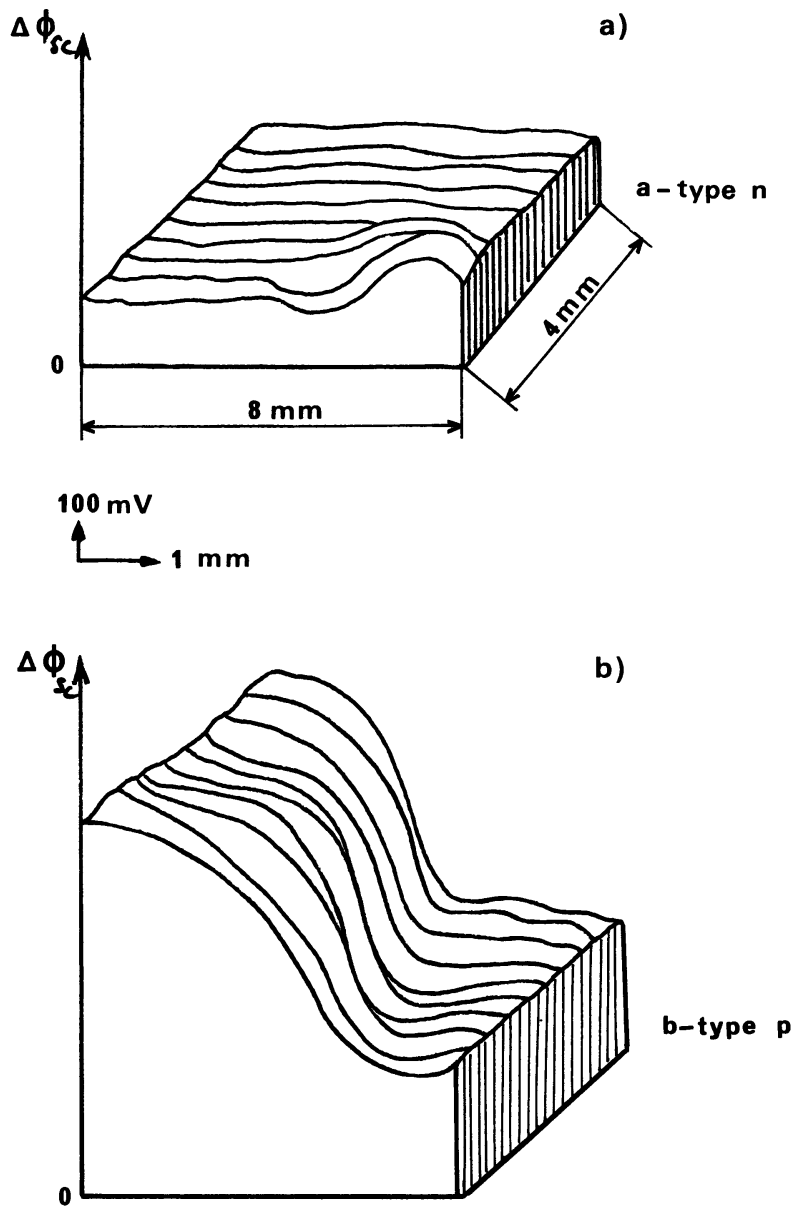

Fig. 2. - Topographies de travail de sortie: $\Delta \phi_{\mathrm{SC}}=$ $\phi_{\mathrm{SC}}-\phi_{\mathrm{M}}, \phi_{\mathrm{M}}=$ travail de sortie de l'électrode de Kelvin.

(a) InP type $n$ : surface (110) obtenue par clivage

(b) InP type $p$ : surface (110) obtenue par clivage

[Work function topographies : $\Delta \phi_{\mathrm{SC}}=\phi_{\mathrm{SC}}-\phi_{\mathrm{M}}, \phi_{\mathrm{M}}=$ work function of the Kelvin probe.

(a) n type InP : surface (110)

(b) p type InP : surface (110)]

clivées des états dus au défaut accepteurs et donneurs de clivage créent une barrière et peuvent conduire à l'ancrage du niveau de Fermi [18, 19].

Cette existence d'états a été également mise en évidence par des méthodes de photoémission [20, 21], de spectroscopie de photovoltage [22].

3.1.2 Etude de l'ancrage du niveau de Fermi. Plaçons-nous dans le cas d'états de surface accepteurs sur un semiconducteur de type $\mathrm{n}$ ou donneurs sur un semiconducteur de type p. Si la densité des états est suffisante, la barrière de surface prend une valeur telle que le niveau de Fermi vient se placer en surface au niveau de l'état [23]. La neutralité du semiconducteur impliquant que la densité de charge en surface $Q_{\mathrm{SS}}$ et la densité de volume $Q_{\mathrm{SC}}$ soient égales, on a donc dans le cas d'accepteurs de densité $N_{\mathrm{a}}$ et de position énergétique $E_{\mathrm{A}}$ :

$$
\begin{aligned}
Q_{\mathrm{SS}}=Q_{\mathrm{SC}} & Q_{\mathrm{SC}}=F\left(V_{\mathrm{s}} \text { dopage }\right)= \\
=- & N_{\mathrm{a}} q\left(1+\exp \frac{E_{\mathrm{A}}-E_{\mathrm{F}}-q V_{\mathrm{s}}}{k T}\right)^{-1} .
\end{aligned}
$$

La détermination de $V_{\mathrm{s}}$ satisfaisant à cette égalité peut être illustrée graphiquement.

La figure 3 représente un réseau de courbes obtenues pour deux dopages différents ; son examen montre que les points d'intersection des deux courbes $F$ avec la courbe $Q_{\mathrm{ss}}\left(V_{\mathrm{s}}\right)$ ont des abscisses $E_{\mathrm{Fs}}$ très voisines. Pour les deux échantillons le niveau de Fermi est venu se fixer au niveau de l'état. Une augmentation de la densité $N_{\mathrm{a}}$ ne modifie guère la position du niveau de Fermi qui est dit ancré en surface. Par contre, à faible valeur de $N_{\mathrm{a}}$, la barrière à l'équilibre sera obtenue alors en considérant l'intersection de la partie horizontale de la courbe $Q_{\text {ss }}$ avec les courbes $F$. Les deux points d'intersection pourront être très éloignés l'un de l'autre. Il n'y a plus d'ancrage.

Des résultats semblables sont obtenus si on considère deux types d'états. Les réseaux de courbes lorsque les états accepteurs sont situés au-dessus des donneurs, puis dans le cas inverse, sont précisés figure 3 .

Lorsque les accepteurs sont situés au-dessous des donneurs, les charges dans chacun des états, dans les mêmes conditions de dopage sont beaucoup plus fortes que lorsque les positions sont inversées. Dans le cas de la figure $3 \mathrm{c}, E_{\mathrm{A}}>E_{\mathrm{D}}$, l'ancrage se fait entre le niveau donneur et le niveau accepteur. Pour le semiconducteur de type n, la variation de $E_{\mathrm{FS}}$ avec $N_{\mathrm{a}}$ et $N_{\mathrm{d}}$ est pratiquement indépendante du rapport $\frac{N_{\mathrm{a}}^{\mathrm{a}}}{N_{\mathrm{d}}}$. Par contre, pour le type $\mathrm{p}$, la position d'ancrage est d'autant plus rapidement atteinte que $\frac{N_{\mathrm{a}}}{N_{\mathrm{d}}}$ est faible. Cette situation est tout à fait différente de celle correspondant à la figure $3 \mathrm{~d}$ pour laquelle l'état donneur est au-dessus de l'état accepteur. L'ancrage ne se fait pas, ici, entre les états mais tout près de l'état donneur si $N_{\mathrm{d}}>N_{\mathrm{a}}$, tout près de l'état accepteur si $N_{\mathrm{a}}>N_{\mathrm{d}}$, entre eux si $N_{\mathrm{a}}=N_{\mathrm{d}}$. Cette différence de comportement s'explique par le fait que lorsque $E_{\mathrm{A}}$ est au-dessous de $E_{\mathrm{D}}$, les états accepteurs sont beaucoup plus chargés que lorsque $E_{\mathrm{A}}>E_{\mathrm{D}}$; la charge de surface nécessaire à l'ancrage résulte alors de la compensation entre deux charges, beaucoup plus élevées que lorsque $E_{\mathrm{A}}>E_{\mathrm{D}}$, mais dont la différence reste du même ordre.

\subsection{DÉPOTS MÉTALLIQUES.}

3.2.1 Modes de croissance. - Lorsque les premiers atomes métalliques arrivent sur la surface, ils peuvent [24] :

- se physisorber et constituer ainsi un état précurseur à une liaison plus énergétique ; 

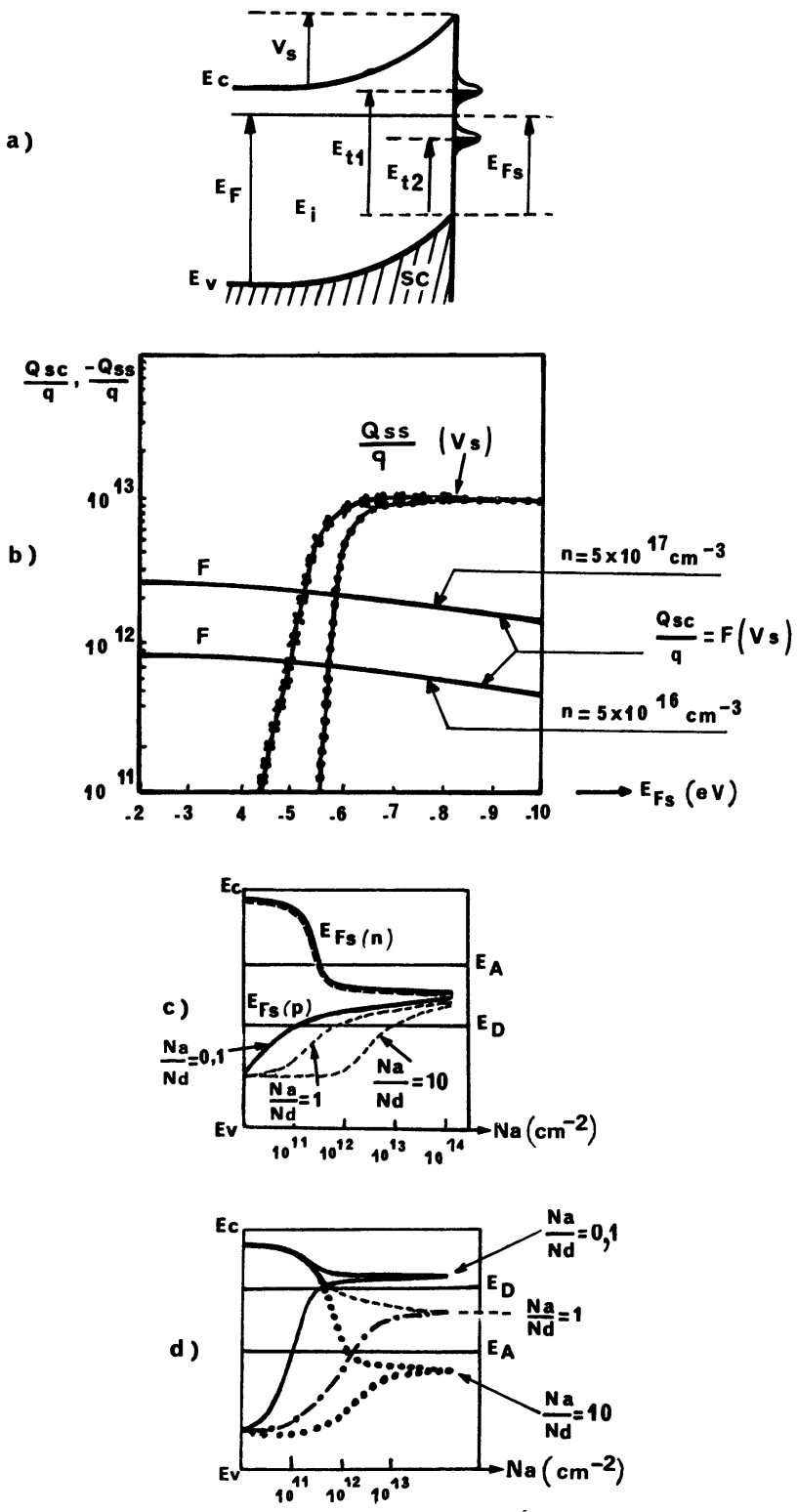

Fig. 3.

(a) Modèle à deux états

(b) Variations de $Q_{\mathrm{SC}}$ et $Q_{\mathrm{Ss}}$ avec $E_{\mathrm{Fs}}$ dans le cas suivant :

- un accepteur (densité $N_{\mathrm{a}}$ ) sur type $\mathrm{n}+++$

- un accepteur (densité $N_{\mathrm{a}}$ ) et un donneur (densité $N_{\mathrm{d}}$ ) sur type n...

(c) (d)Variation de la position de $E_{\mathrm{Fs}}$ en présence d'un état donneur et d'un état accepteur en fonction de la densité des états accepteurs $N_{\mathrm{a}}$ et donneurs $N_{\mathrm{d}}$ pour $\frac{N_{\mathrm{a}}}{N_{\mathrm{d}}}=$ constante $10,1,0,1 . c=E_{\mathrm{A}}>E_{\mathrm{D}}-d=$ $E_{\mathrm{D}}>E_{\mathrm{A}}$

[(a) Two state model

(b) $Q_{\mathrm{SC}}$ and $Q_{\mathrm{SS}}$ versus $E_{\mathrm{Fs}}$ variations

- acceptor surface states (density $N_{\mathrm{a}}$ ) on $\mathrm{n}$ type $+++$

- acceptor surface states (density $N_{\mathrm{a}}$ ) and donor (density $N_{\mathrm{d}}$ ) surface states on $\mathrm{n}$ type...

(c) (d) $E_{\mathrm{Fs}}$ variation versus $\frac{N_{\mathrm{a}}}{N_{\mathrm{d}}}$ for $E_{\mathrm{A}}>E_{\mathrm{D}}$ and $E_{\mathrm{D}}>$ $\left.E_{\mathrm{A}}\right]$
- s'ionosorber, c'est-à-dire se transformer en ion par échange avec la population de porteurs libres. Dans ce cas, ils ont un comportement électronique identique à celui d'un état et l'on peut leur associer un niveau ;

- se fixer sur un site par des liaisons locales.

Ils peuvent ensuite diffuser le long de la surface, rencontrer d'autres atomes mobiles et former avec eux des amas qui se stabiliseront, grossiront et coalesceront sous forme de cristallites ou s'agglomérer à des cristallites en cours de croissance qui en se développant feront le film continu... ou désorber.

La croissance du film ainsi schématisée peut s'effectuer suivant différents modes liés à la nature des liaisons interfaciales, à l'énergie de cohésion des métaux et des semiconducteurs, à la nature de la surface et aux propriétés de diffusion du métal... Partant de considérations thermodynamiques et de données expérimentales Bauer [25] a proposé les trois modes schématisés figure 4.

Dans la croissance suivant le mode VolmerWeber, caractéristique de systèmes présentant des paramètres de maille différents et une énergie libre de surface $\left(E_{\mathrm{S}}\right)$ et d'interface $\left(E_{\mathrm{i}}\right)$ telle que $E_{\mathrm{S}}>E_{\mathrm{i}} / 2$, les atomes diffusent sur la surface et se regroupent en îlots : la croissance est une croissance par îlots.

Le modèle de Frank-Van der Merwe qui correspond à une croissance couche par couche est obtenu quand le substrat et le film ont des paramètres de réseau voisin et quand la liaison substrat-adsorbat est plus forte que la liaison adsorbat-adsorbat, c'està-dire quand $E_{\mathrm{i}}>E_{\mathrm{S}} / 2$.

Enfin, le troisième mode dit Stranski-Krastanov est une synthèse des deux. Les paramètres de réseau sont différents mais $E_{\mathrm{S}}<E_{\mathrm{i}} / 2$. On a alors croissance d'une première couche ce qui implique une forte interaction substrat atome adsorbé, suivie d'une croissance par îlots.

L'étude de ces modes de croissance est effectuée, in situ, en s'appuyant, en particulier, sur une étude Auger fine des pics, sur un suivi en diffraction d'électrons lents ou diffraction d'électrons de haute énergie, en spectroscopie de photoémission dans l'ultraviolet... [26, 27] et ex situ par microscopie électronique à transmission [28]. Elle montre que sur les III-V, en particulier, si la croissance se fait plus fréquemment suivant le mode Stranski-Krastanov (Al, In, Ga sur InP (110), Al, sur GaAs 100), elle se fait également suivant le mode Frank-Van der Merwe [ $\alpha$-Sn sur InSb (100) et CdTe (100), Sb sur InSb (111)] et Volmer-Weber [Au, Ag sur GaAs (110) (100)].

3.2.2 Formation et structure de l'interface. Lorsqu'on dépose le métal, les propriétés de la surface de départ, puis de l'interface en cours de formation évoluent jusqu'à ce que l'interface puisse 

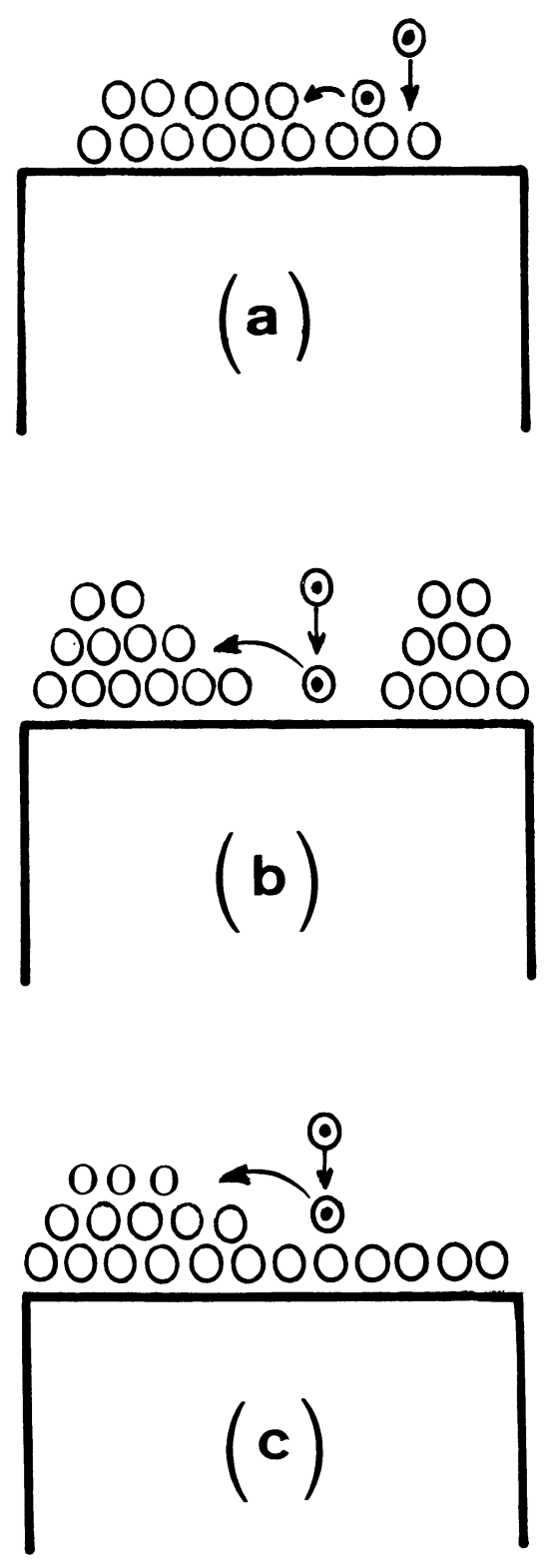

$\odot$ atome incident

$O$ atome fixe

Fig. 4. - Modes de croissance du métal : (a) Franck Van der Merwe (b) Volmer Weber (c) Stranski-Krastanov.

[Crystal growth modes: (a) Franck Van der Merwe (b) Volmer Weber (c) Stranski-Krastanov.]

être considérée comme stable. Cette évolution se fait en plusieurs phases schématisables comme suit.

La première phase correspond aux tout premiers dépôts, c'est-à-dire à un recouvrement inférieur à la demi-monocouche. Elle est appelée parfois phase diluée car la quantité de métal est trop faible pour qu'on puisse avoir un film métallique continu en surface. Les caractéristiques dépendent de l'interaction directe entre atomes et sites de surface au travers de données thermodynamiques comme la chaleur de condensation du métal, de cohésion du semiconducteur, mais aussi d'autres quantités comme la vitesse de dépôt, les températures. Son importance est grande car elle induit déjà l'ancrage du niveau de Fermi ; de plus, la diffusion commence déjà dès les premiers recouvrements.

La deuxième étape correspond en gros à la formation de la première monocouche voire de la deuxième. La quantité de métal déposé est encore insuffisante pour que le dépôt présente des propriétés métalliques $[29,30]$ et les propriétés de l'interface ainsi élaborée sont très dépendantes de la structure de la couche métallique et par la même de la température de dépôt et de traitements thermiques éventuels. Les effets de diffusion, d'électromigration, les réactions sont généralement importantes durant cette deuxième phase.

Les dépôts suivants contribuent encore à la formation de l'interface. L'évolution des contraintes dues au désaccord de réseau, la présence de joints de grains font que l'interface n'atteint une configuration quasi stable que lorsque le dépôt atteint plusieurs monocouches ( 3 à 10 pour GaAs). Cette stabilité n'est d'ailleurs que relative car des diffusions lentes à la température ambiante ou accélérées par traitement thermique peuvent encore la faire évoluer.

3.2.3 Etude des propriétés de l'interface en cours de croissance. - Nous donnerons seulement ici quelques résultats de façon à ne pas alourdir la présentation.

La figure 5 (31) représente la variation du travail de sortie obtenue par la méthode de Kelvin lors de dépôt d'Au, Ag, Al sur InP (110). Elle montre que dans ces trois cas, les travaux de sortie des échantillons de type $\mathrm{p}$ et $\mathrm{n}$ sont déjà pratiquement égaux, pour les dépôts de l'ordre de 1 monocouche; le métal créé des défauts donneurs et accepteurs en densité suffisante pour que l'ancrage soit très rapide. Les variations de $\phi_{\text {SC }}$ qu'on obtient ensuite lorsqu'on augmente la quantité de métal déposé sont dues à des modifications de l'affinité électronique au travers du dipôle semiconducteur-métal.

Cette méthode présente l'inconvénient de ne pas permettre à tout instant de séparer sans ambiguïté les variations de la barrière de celles de l'affinité électronique. On est conduit à y associer des mesures de photovoltage dont on peut moyennant certaines hypothèses (comme l'a fait Brillson [9] ou comme nous l'avons fait nous-mêmes [31]) déduire les barrières. Il faut cependant être prudent ; en particulier, la simplification qui consiste à considérer que dans des conditions d'éclairement classiques, on arrive aux bandes plates, est irréaliste.

Ces réserves étant faites, la méthode présente des particularités très intéressantes : elle n'est pas perturbatrice et elle permet de mettre en évidence, au 


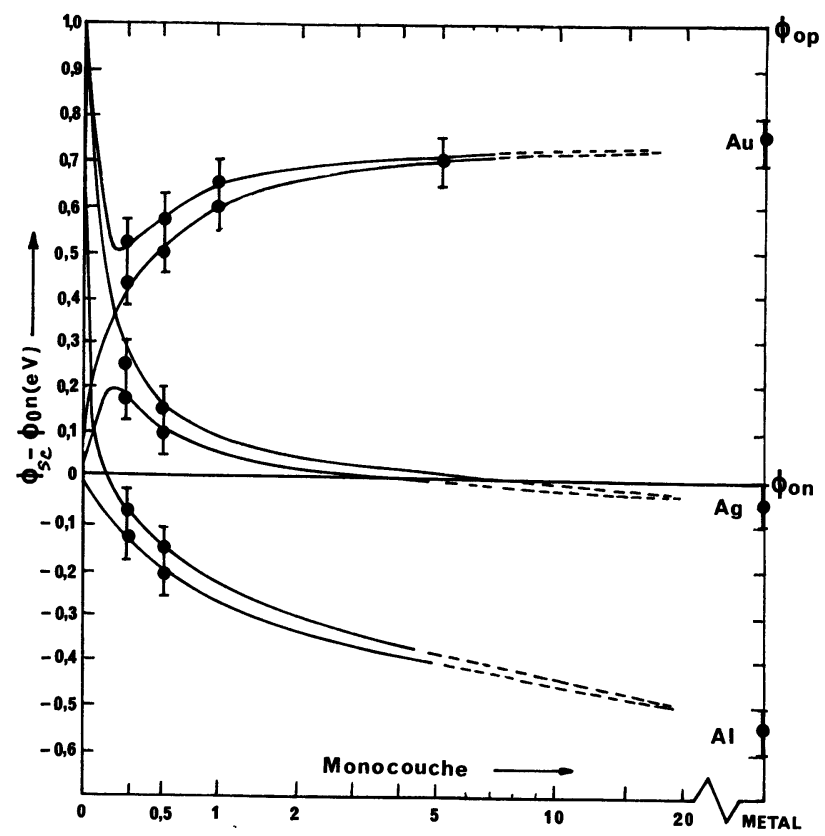

Fig. 5. - Variations du travail de sortie d'échantillons clivés d'InP type $n$ et type $p$ avec le recouvrement avec $\mathrm{Au}, \mathrm{Ag}, \mathrm{Al}$.

[Work function variations of $\mathrm{n}$ and $\mathrm{p}$ type InP (110) surfaces versus $\mathrm{Au}, \mathrm{Ag}, \mathrm{Al}$ coverage.]

travers de la barrière de surface, des états chargés négativement ainsi que des états chargés positivement. De plus, moyennant une modélisation, elle permet de situer la position des états. Ce n'est cependant pas une méthode spectroscopique, comme la photoémission par exemple. Celle-ci permet en effet de localiser directement dans la bande interdite des états occupés et d'en suivre l'évolution.

La figurẹ 6 obtenue dans le groupe dirigé par C. Sebenne [32], utilisant des énergies de photons voisines du seuil de photoémission, en illustre les possibilités. La densité des états, déjà mis en évidence pour des recouvrements $\theta$ aussi faibles que quelque $10^{-3}$ monocouche croît ensuite régulièrement avec $\theta$.

L'utilisation d'énergies plus élevées correspondant à l'ultraviolet (quelque $10 \mathrm{eV}$, UPS), aux rayons X (1 $254 \mathrm{eV}-\mathrm{Mg}, 1454 \mathrm{Al}$ XPS) fournit beaucoup de renseignements sur la bande de valence, les niveaux de cour et donc sur l'interaction chimique. Le rayonnement synchrotron a permis, en particulier, une avancée remarquable dans ce domaine [33, 34, 63]. Outre les résultats sur la structure de bande en volume, les utilisateurs ont pu en déduire des propriétés des états de surface, de liaisons pendantes, des énergies de liaison, de la distribution de charge de surface... C'est donc un outil très puissant.

D'autres méthodes complémentaires jouent un rôle essentiel dans le suivi de la formation de l'interface.

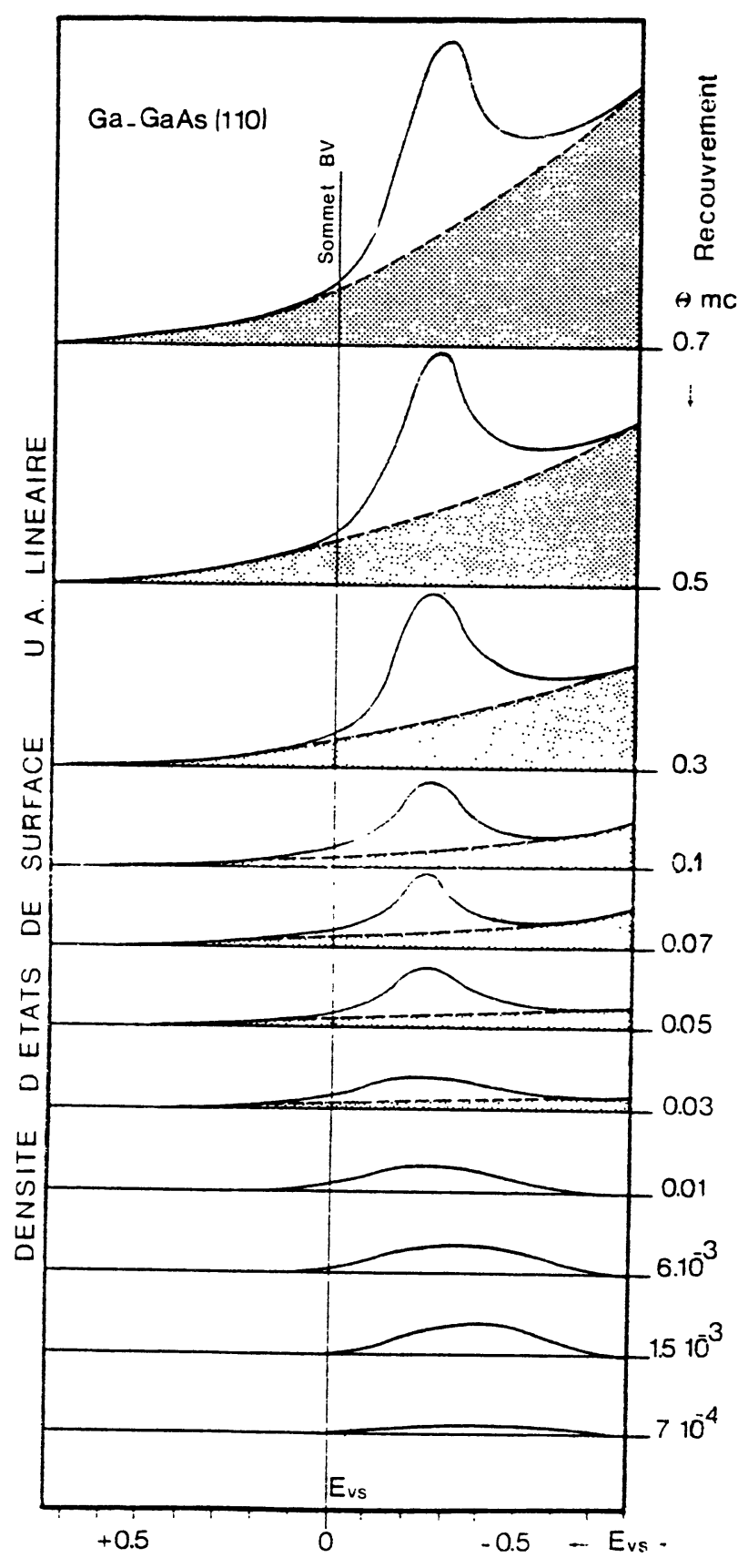

Fig. 6. - Densité d'états sur GaAs en fonction du recouvrement en Ga (Réf. 32).

[Density of states on GaAs versus Ga coverage (Réf. [32]).]

\section{C'est le cas de :}

- l'effet Auger qui permet le contrôle de la surface, le suivi de son évolution et de sa stœchiométrie, l'étude de la croissance du film métallique et de la physicochimie de l'interface lors de l'interaction; - de la diffraction d'électrons lents (DEL) ou rapides (RHEED) qui apporte une information importante sur la structure de la surface initiale et celle du dépôt ;

- de la mesure des pertes d'électrons qui renseigne sur les phonons ou plasmons de surface et sur les 
transitions électroniques d'électrons de liaison des atomes de surface vers des états vides situés audessus du niveau de Fermi. D'autres méthodes comme l'effet Raman, la retrodiffusion d'ions... etc., ont été également utilisées mais de façon ponctuelle.

3.3 INTERFACE FINIE. - Lorsque la couche métallique dépasse plusieurs dizaines d'angströms l'interface peut être considérée comme finie et ce bien qu'elle puisse cependant encore être sujette à des évolutions en particulier à cause de diffusion, de dissociation, de liaisons. Ces interfaces qui définissent les propriétés des diodes, propriétés que l'on obtient par des mesures I(V), C(V) [35-37] ou de photoréponse peuvent être schématisées par une présentation générale faisant intervenir les différentes zones suivantes (Fig. 7) :

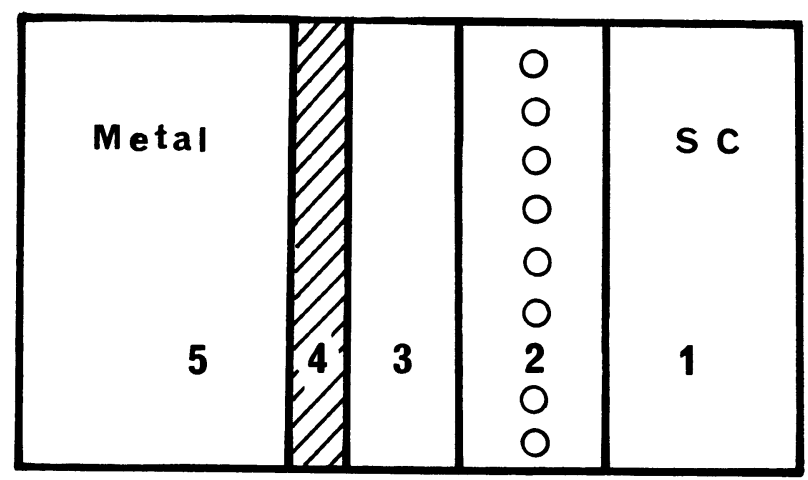

Fig. 7. - Structure de l'interface : Zone 1 Semiconducteur non perturbé; Zone 2 Semiconducteur avec défauts induits par la diffusion ; Zone 3 Composé nouveau éventuel ; Zone 4 Zone métallique perturbée ; Zone 5 Métal.

[Interface structure : Zone 1 Undisturbed semiconductor ; Zone 2 Semiconductor with defects ; Zone 3 New compound ; Zone 4 Disturbed metal ; Zone 5 Metal.]

zone 1: semiconducteur non perturbé dans sa stœchiométrie, ni dans ses états électroniques ;

zone 2 : semiconducteur perturbé par des atomes métalliques diffusés, par des lacunes résultant de l'exodiffusion de ses constituants, par des défauts extrinsèques induits par le métal ;

zone 3 : une zone intermédiaire correspondant à

la formation éventuelle d'un composé nouveau ;

zone 4 : une zone de faible épaisseur correspondant au métal perturbé et par exemple à la formation d'un alliage ;

zone 5 : le métal proprement dit.

Cette subdivision peut encore être précisée si on prend en compte les états induits par le métal dont l'extension coté semiconducteur est de l'ordre de $10 \AA$. Suivant les dimensions des zones 3 et 2 , on pourra donc être amené à y délimiter des régions avec états métalliques et des régions sans états métalliques.

L'importance de la zone de transition entre métal et semiconducteur comme celle des différentes zones ci-dessus dépend de la nature des matériaux en présence et des traitements subis. Suivant la chaleur de réaction à l'interface l'étendue pourra aller de quelques $\AA$ à quelques dizaines d' $\AA$.

Dans le cas du silicium et de métaux nobles, cette zone peut être très étendue et aller jusqu'à écranter complètement l'interface siliciure-semiconducteur de l'action du métal de l'électrode.

\subsection{QUELQUeS RÉSUltats. - Nous présentons} (Fig. $8 \mathrm{a}, \mathrm{b}, \mathrm{c}$ ) quelques résultats obtenus au LESIC qui montrent l'évolution de l'ancrage du niveau de Fermi sur InP au cours des étapes précédentes, ainsi que quelques caractéristiques essentielles des diodes obtenues sur GaAs et InP (Tab. Ia, b, et II).

L'analyse de ces résultats montre tout d'abord que l'ancrage du niveau de Fermi pour GaAs se fait toujours dans la partie inférieure de la bande interdite et pour InP dans la partie supérieure. Si la nature du métal ne modifie pas de façon très importante cette position de l'ancrage, elle se traduit cependant par des légères variations dans la barrière de surface, en particulier dans le cas d'InP pour lequel les métaux réactifs conduisent à un ancrage beaucoup plus proche de la bande de conduction que les métaux non réactifs.

On note de plus une similitude entre les résultats obtenus par le dépôt de quelques monocouches et ceux déduits de l'étude des diodes.

Enfin, une perturbation de l'interface induite par adsorption d'oxygène ou dépôt d'une couche intercalaire entraîne un changement sensible dans l'ancrage et la barrière dans les diodes.

En ce qui concerne ces dernières, les valeurs numériques données dans les tableaux montrent que le coefficient d'idéalité, pour des diodes élaborées simultanément sur un même substrat, peut varier de façon très sensible. Même dans le cas des diodes élaborées sur surfaces clivées, on ne peut être certain de la qualité «électronique » de l'interface.

\section{Modèles.}

La complexité du problème rejaillit sur la multiplicité des modèles. Certains d'entre eux, les plus anciens sont basés sur des mécanismes physiques qui rendent compte macroscopiquement des effets observés. Ils ne se sont pas attachés à la recherche exhaustive des causes physiques, de la nature possible de ces mécanismes. Les modèles les plus récents sont, à l'inverse, focalisés sur les origines physiques. 


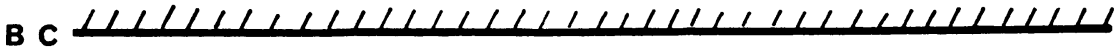

a)

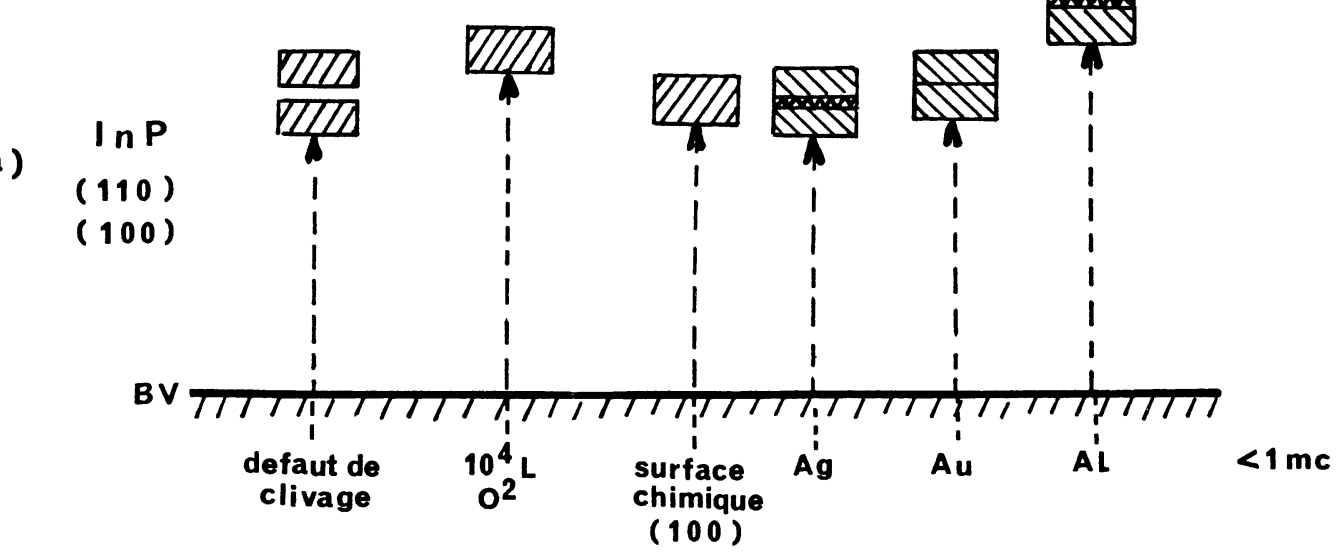

b )

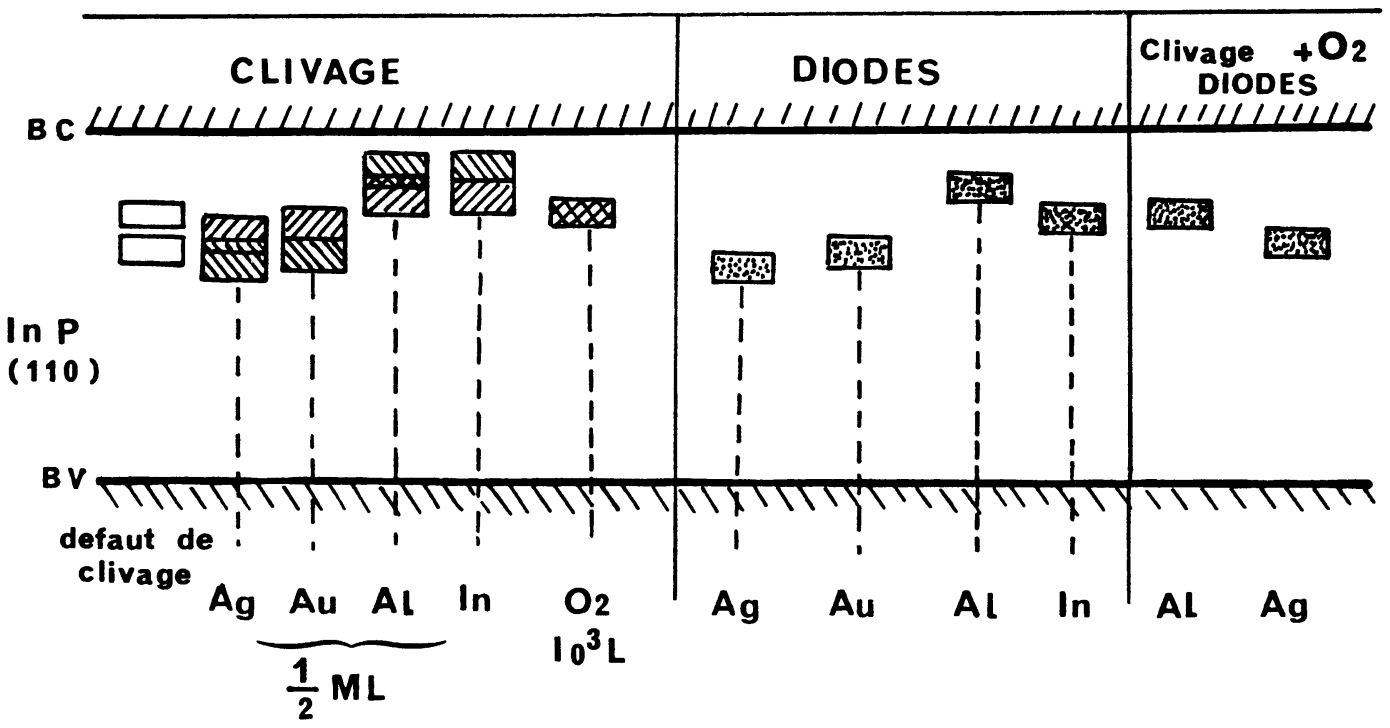

BC

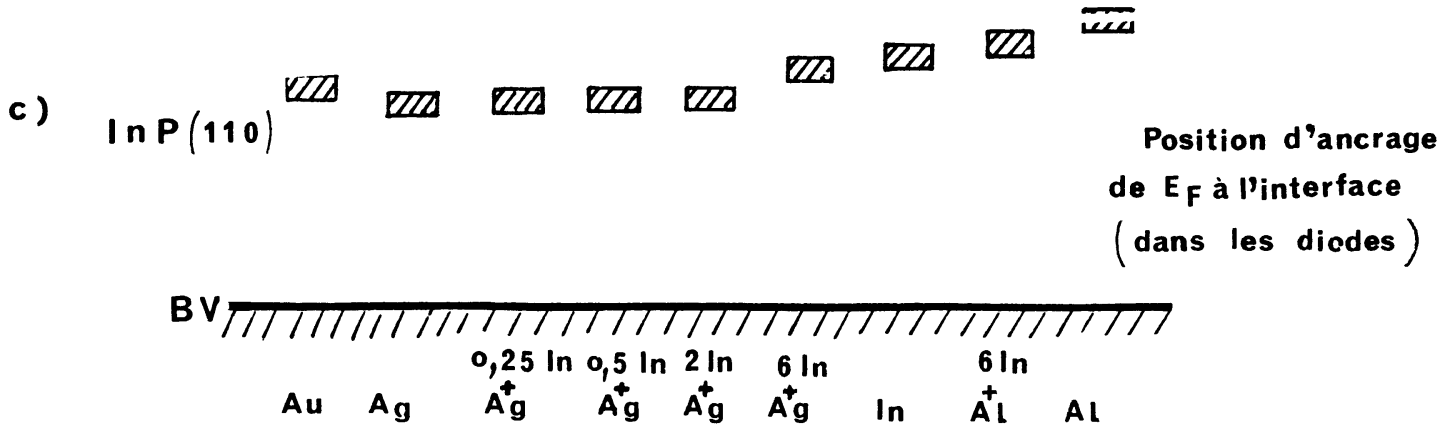

Fig. 8. - Ancrage du niveau de Fermi dans la bande interdite de $\operatorname{InP}(110)$ : (a) en surface (b) en surface et dans les diodes (c) contribution d'une couche interfaciale d'indium.

[Fermi level pinning on (110) InP surfaces and in Schottky diodes : (a) surface Fermi level pinning (b) surface Fermi level pinning on (110) surfaces and in the diodes (c) contribution of an interlayer to the Fermi level pinning.] 
Tableaux Ia-Ib Diodes élaborées au LESIC.

[Diodes achieved by the LESIC.]

Tableau Ia. - Barrière de Schottky à $300 \mathrm{~K}$ sur surfaces clivées (110) et sur surfaces obtenues par nettoyage chimique. Les valeurs $n_{\max }$ et $n_{\min } n$ sont les valeurs limites ou moyennes obtenues sur des diodes élaborées en même temps, sur une même plaquette. $\mathrm{N}_{1}$ et $\mathrm{N}_{2}$ correspondent à deux nettoyages chimiques différents. Les chiffres qui leur sont associés ont pour objet d'illustrer une variation que peut induire un nettoyage.

[Schottky barriers at $300 \mathrm{~K}$ on (110) cleaved and (100) etched GaAs surfaces. $n_{\max }, n_{\min } n$ correspond to the maximum, the minimum, the mean values of $n$ for diodes simultaneously elaborated on the same substrate. $\mathrm{N}_{1}, \mathrm{~N}_{2}$ correspond to two different and classical processes for chemical etching.]

\begin{tabular}{|c|c|c|c|c|c|c|c|c|}
\hline & \multirow{3}{*}{$\begin{array}{l}\sigma_{B} l(v) \quad(m e V) \\
\alpha_{B} c(v) \quad(m e V)\end{array}$} & $\begin{array}{c}\text { GaAs }(100)+A g \\
N 1\end{array}$ & $\begin{array}{c}\text { GaAs }(100)+A g \\
N_{2}\end{array}$ & $\begin{array}{c}\text { GaAs (100)+AI } \\
\text { N2 }\end{array}$ & $\begin{array}{c}\text { GaAs }(100)+A u \\
\text { N } 2\end{array}$ & $\begin{array}{c}\text { GaAs clivé } \\
+\mathrm{Ag}\end{array}$ & $\begin{array}{c}\text { GaAs clivé } \\
+\mathrm{Au}\end{array}$ & $\begin{array}{c}\text { GaAs clivé } \\
+\mathrm{Al}\end{array}$ \\
\hline & & 360 & 410 & 724 & 446 & 375 & 410 & 600 \\
\hline 0 & & & & 808 & & & & 630 \\
\hline & $n_{\text {mire }}^{n} n_{\max }$ ou & 1.9 & $1.53-1.96$ & $1.13-1.16$ & $1.18-2.55$ & $1.2-1.8$ & $1.20-1.30$ & $1.04-1.15$ \\
\hline & $\alpha_{B} I(v) \quad(m c v)$ & 904 & 823 & 784 & 840 & 820 & 880 & 700 \\
\hline 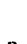 & $\alpha_{B} c(v) \quad(m e V)$ & 1093 & 944 & 807 & 966 & 1030 & 980 & 740 \\
\hline & $n_{\min }, n_{\max }$ & $1.09-1.36$ & $1.04-1.15$ & $1.13-1.25$ & $1.07-1.11$ & $1.11-1.18$ & $1.1-1.14$ & $1.07-1.11$ \\
\hline
\end{tabular}

Tableau Ib. - Barrière de Schottky et coefficient' d'idéalité à $300 \mathrm{~K}$ sur InP (110).

[Schottky barriers and ideality factor at $300 \mathrm{~K}$ on InP (110).]

\begin{tabular}{|c|c|c|c|c|c|}
\hline & & $\operatorname{In} P(110)+A g$ & $\operatorname{InP}(110)+A u$ & $\operatorname{In} P(110)+\operatorname{In}$ & $\ln P(110)+A L$ \\
\hline Type $p$ & $\begin{array}{l}\alpha_{B} I(v) \quad(m e V) \\
n \\
\alpha_{B} c(v)(m e V)\end{array}$ & $\begin{array}{l}780 \\
1.10 \\
850\end{array}$ & $\begin{array}{l}800 \\
1.40 \\
990\end{array}$ & $\begin{array}{l}890 \\
1.1\end{array}$ & $\begin{array}{c}1000 \\
1.15 \\
1370\end{array}$ \\
\hline Type $n$ & ${ }_{n}^{\alpha_{B} I(v) \quad(m e v)}$ & $\begin{array}{l}515 \\
1.20\end{array}$ & $\begin{array}{l}430 \\
1.02\end{array}$ & $\begin{array}{l}350 \\
1.5\end{array}$ & ohmique \\
\hline
\end{tabular}

Tableau II. - Barrières de Schottky sur InP(n) et GaAs(n) obtenues à $300 \mathrm{~K}$ (Réf. [36]).

$\mathrm{P}=$ Atomiquement propre

$\mathrm{C}=$ Nettoyage chimique

[Schottky barriers and ideality factor for InP and GaAs at $300 \mathrm{~K}$ (Ref. [36]).

$\mathbf{P}=$ Atomically clean

$\mathrm{C}=$ Chemically etched.]

\begin{tabular}{|c|c|c|c|c|c|c|c|c|}
\hline METAL & \multicolumn{2}{|c|}{$\mathrm{Pt}_{\mathrm{t}}$} & \multicolumn{2}{|c|}{$\mathrm{Cu}$} & \multicolumn{2}{|c|}{$w$} & $\mathrm{Ni}$ & In \\
\hline & $\alpha_{B} I(v)$ & $\alpha_{B} C(v)$ & $\alpha_{B} I(v)$ & $a_{B} c(v)$ & $\sigma_{B} I(v)$ & $\alpha_{B} c(v)$ & $\alpha_{B} I(v) \quad \alpha_{B} c(v)$ & $\alpha_{B} I(v)$ \\
\hline $\begin{array}{r}\text { GaAs }(110) \mathrm{P} \\
(100) \mathrm{C} \\
(111) \mathrm{C}\end{array}$ & $\begin{array}{ll} & 0.84 \\
\text { à } & 0.92\end{array}$ & 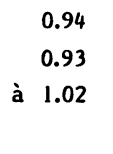 & $\begin{array}{l}0.73 \\
0.80\end{array}$ & 0.87 & $\begin{array}{l}0.71 \\
0.65-0.73\end{array}$ & $\begin{array}{l}0.77 \\
0.69\end{array}$ & $\begin{array}{l}0.79 \\
0.70-0.83 \\
0.67-0.90\end{array}$ & $0.76-0.91$ \\
\hline $\begin{array}{rr}\ln P \quad(110) P \\
\\
(100) C\end{array}$ & 0.52 & 0.54 & & 0.49 & & & $\begin{array}{l}\text { ohmique } \\
0.36\end{array}$ & ohmique \\
\hline
\end{tabular}




\section{1 . MODÈLES DE BASE.}

4.1.1 Modèle de Schottky. - Les premières interprétations des barrières de Schottky reposaient sur le modèle de Schottky dont on a vu qu'il suppose que le contact entre le métal et le semiconducteur se traduit seulement par un transfert de charge. La chute de potentiel qui apparaît aux bornes de la zone de déplétion ainsi formée dans le semiconducteur s'ajuste de façon à égaler les travaux de sortie des deux matériaux. On a donc

$$
-q V_{\mathrm{s}}=\phi_{\mathrm{M}}-\phi_{\mathrm{SC}} \text {. }
$$

Ce modèle ne rend pas compte de la plupart des contacts Schottky sur les III-V et sur les matériaux de la quatrième colonne du tableau de Mendeleieff.

4.1.2 Modèle de Bardeen. - Ce modèle rend compte de la présence d'états d'interfaces qui écrantent l'action du métal et font que la hauteur de barrière reste indépendante de son travail de sortie. La nécessaire différence entre le travail de sortie du métal et celui, fixé par les états, du semiconducteur est alors assurée par un dipôle d'interface, ce qui suppose que les états ne soient pas «en contact direct » avec le métal, car celui-ci écranterait leur action, voire les ferait disparaître. Ceci implique soit que la distance métal-semiconducteur soit supérieure à $3 \AA$ environ, soit que les états soient spatialement distribués dans le semiconducteur.

4.1.3 Modèle de Cowley et Sze. - Les deux modèles précédents sont des cas limites d'un modèle plus général, le modèle de Cowley et Sze. Dans ce modèle, le semiconducteur et le métal sont séparés par une couche isolante d'épaisseur $\delta$. La barrière de Schottky s'exprime alors en fonction du travail de sortie $\phi_{M}$ à l'aide de la relation $\phi_{\mathrm{B}}$ (dans le cas où les bandes sont plates) $=C_{1} \phi_{\mathrm{M}}+C_{2}$ où $C_{1}$ est donné en fonction de la constante diélectrique $\varepsilon$ de la couche et de la densité d'états d'interfaces par la relation :

$$
C_{1}=\frac{\varepsilon}{\varepsilon+q^{2} \delta D_{\mathrm{S}}}
$$

Lorsque la densité $D_{\mathrm{S}}$ est suffisamment élevée pour que $q^{2} D_{\mathrm{S}} \delta \gg \varepsilon$ alors on retrouve le modèle de Bardeen et une barrière indépendante du métal. Inversement si $D_{\mathrm{S}}$ tend vers $0 \frac{\mathrm{d} \phi_{\mathrm{B}}}{\mathrm{d} \phi_{\mathrm{M}}}=1$; on retrouve le modèle de Schottky. Ce modèle de Cowley et Sze est particulièrement adapté au cas des diodes Schottky réalisées par dépôt métallique sur surface réelle.

4.2 DÉVELOPPEMENTS RÉCENTS. - Les modèles précédents cernent des mécanismes qui permettent d'expliquer les résultats expérimentaux. Le modèle de Bardeen basé sur l'ancrage du niveau de Fermi par des états de surface permet d'expliquer de nombreux résultats expérimentaux à partir de distributions simples d'états. L'origine physique de ces états dont la densité nécessaire à l'ancrage peut ne pas excéder le $1 / 100$ de monocouche n'est pas élucidée. Les résultats expérimentaux montrent simplement que ces états semblent être indépendants de la nature du métal. Ceci a conduit à proposer différentes origines à ces états.

4.2.1 Modèle des états intrinsèques: interface abrupte. - Les surfaces (110) relaxées, ne présentent pas d'états dans la bande interdite, exception faite de $\mathrm{GaP}$ pour qui une bande vide d'états de surface se trouverait dans la partie supérieure de la bande interdite, près de la bande de conduction. L'ancrage à l'interface, comme dans les diodes, ne peut être attribué à ces états sauf si on suppose que l'interaction avec le métal les ramène dans la bande interdite. Certains auteurs ont exclu cette éventualité en se basant sur des études fines de photoémission angulaire [38].

D'autres, par contre, considèrent cette hypothèse comme possible [39]. La controverse n'est pas levée sur ce point comme nous le verrons dans un prochain paragraphe.

4.2.2 Modèle des défauts de Spicer [40-41]. -Les arguments précédents ajoutés au fait que, d'une part, sur les III-V l'ancrage du niveau de Fermi se faisait, quelle que soit la nature du métal, au même endroit dans la bande interdite et que, d'autre part cet ancrage était obtenu pour des densités d'atomes métalliques de l'ordre de $10^{12} \mathrm{~cm}^{-2}$ seulement ont conduit Spicer [41] à mettre en cause des défauts induits par le métal. Les atomes du métal en se condensant sur la surface, libéreraient une énergie suffisante pour créer des défauts spécifiques du semiconducteur, indépendants de la nature du métal. Dans l'hypothèse de Spicer, ces défauts seraient des lacunes du cation qui se comporteraient comme des donneurs et des lacunes de l'anion qui se comporteraient comme des accepteurs. Les états correspondants, caractéristiques de chaque semiconducteur, seraient localisés comme précisé figure 9.

A faible recouvrement, ce modèle traduit bien de nombreux résultats expérimentaux, en particulier si on envisage que le taux de création des défauts est lié à la nature du métal déposé. Les barrières obtenues par l'étude des diodes et la position de l'ancrage à faible recouvrement évoluent globalement de façon voisine, mais les différences notées dans certains cas, ajoutées au fait que la précision des mesures n'est pas très grande, font qu'on ne peut conclure sans ambiguïté que la position des états est unique, indépendante de la nature de l'interaction de surface.

Dans l'état, cependant, ce modèle constitue un pas de plus dans l'appréhension de la physique des interfaces et d'autres groupes se sont attachés dans 
Ga As
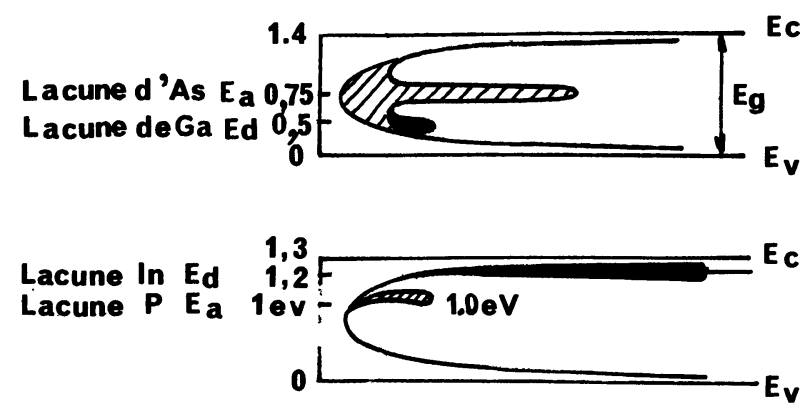

$\ln \mathbf{P}$

Fig. 9. - Modèle des défauts induits de Spicer : Distribution des états de surface pour GaAs, InP (110).

[Spicer Model : Defect induced surface states for GaAs and InP (110) surfaces.]

l'optique précédente, à rechercher des causes physiques, autres que les lacunes, susceptibles de rendre compte des niveaux et des effets obtenus.

C'est ainsi que Allen et Dow [42-44] ont supposé que les défauts résultaient du fait que certains atomes étaient en position antisite (As à la place de $\mathrm{Ga}=\mathrm{As}_{\mathrm{Ga}}$ ou $\mathrm{Ga}$ à la place d'As $=\mathrm{Ga}_{\mathrm{As}}$ ) et ils ont calculé les positions énergétiques des états associés à ces défauts. Ils ont ensuite expliqué à partir de là de nombreux résultats expérimentaux et, en particulier, ceux de Williams [34] qui avait conclu pour InP à l'existence de deux barrières, l'une associée aux métaux réactifs, l'autre, aux métaux non réactifs. L'association d'antisites aux métaux non réactifs, de lacunes aux métaux réactifs conduit à un bon accord entre la théorie et les expériences de Williams.

J. D. Dow et al. [44] ont ensuite prédit les barrières de Schottky sur un certain nombre de semiconducteurs. La comparaison avec les résultats expérimentaux est satisfaisante, en particulier, si on regarde les variations de barrière avec la composition dans les alliages. Elle l'est moins si on s'intéresse aux valeurs de la barrière. Cela peut s'expliquer cependant si on prend en compte la marge d'erreur de l'évaluation théorique.

4.2.3 Etats induits par le métal. - Une autre origine possible a été envisagée pour ces états. Heine [45] a envisagé que des électrons du métal puissent par effet tunnel passer dans la bande interdite du semiconducteur. Les fonctions d'ondes associées à ces électrons sont caractérisées par une longueur d'atténuation de l'ordre de $10 \AA ̊$. Les états correspondants se substituent, en fait, aux états induits par la rupture initiale du réseau cristallin.

Une école s'est développée autour de cette hypothèse [46, 47]. Pellegrini a effectué des calculs sur les modifications de barrière dans les diodes résultant des états de Heine. Inkson a proposé un modèle d'ancrage basé sur la réduction de la bande interdite du semiconducteur lorsqu'on se rapproche de l'interface, réduction allant jusqu'à un gap 0 au contact.

Plus tard, Louie et al. [48-50] ont effectué des calculs à partir d'un modèle associant le métal à un jellium. Ils ont calculé la redistribution des charges à l'interface et mis en évidence des états non discrets qui occupent une partie de la bande interdite et qui décroissent exponentiellement de part et d'autre de l'interface.

Dans cette optique, Tersoff [51,52] a récemment reconsidéré le problème. Partant de l'existence d'états induits par le métal dans la bande interdite, de densité suffisante pour écranter sur quelques $\AA$, toute modification locale de charge, Tersoff définit un niveau $E_{\mathrm{B}}$, déterminé par la distribution dans la bande interdite en surface des états dérivant de la bande de valence et de la bande de conduction du semiconducteur. C'est sur ou près de ce niveau $E_{\mathrm{B}}$ qui correspond à la neutralité locale que vient se placer le niveau de Fermi. Connaissant $E_{\mathrm{B}}$, on en déduit donc que la barrière de Schottky est déterminée essentiellement par les propriétés du semiconducteur, le métal et les particularités de l'interface intervenant au second ordre. Les résultats déduits du calcul et les résultats expérimentaux sont tout aussi comparables que dans les cas précédents, comme le montre le tableau III ci-dessous qui donne les valeurs théoriques de $E_{\mathrm{B}}$, la marge de variation expérimentale de $\phi_{\mathrm{B}}$ pour $\mathrm{Si}$ et $\mathrm{GaAs}$

$$
\begin{array}{lccr} 
& E_{\mathrm{g}} & E_{\mathrm{B}} & \phi_{\mathrm{B}} \\
\mathrm{Si} & 1,12 & 0,76 & 0,70 \rightarrow 0,82 \\
\text { GaAs } & 1,42 & 0,74 & 0,71 \rightarrow 0,94
\end{array}
$$

Des calculs dans le même esprit ont été développés par M. Lannoo et al. [53, 54]. Les propriétés électroniques de l'interface sont déterminées par le couplage entre les liaisons pendantes de surface du semiconducteur et les états du métal. Le calcul de la position du niveau de Fermi $E_{\mathrm{F}}$ en surface montre alors que dans le cas des III-V, $E_{\mathrm{F}}$ vient s'aligner avec un niveau $E_{\mathrm{d}}$ qui est lié aux niveaux énergétiques des liaisons pendantes de l'anion $E_{\mathrm{A}}$ et du cation $E_{\mathrm{B}}$ par la relation simple

$$
E_{\mathrm{d}}=\frac{E_{\mathrm{A}}+E_{\mathrm{B}}}{2}
$$

$E_{\mathrm{d}}$ joue le même rôle que $E_{\mathrm{B}}$ dans la théorie de Tersoff.

4.2.4 Modèle de Brillson [9]. - Ce modèle est envisageable quand l'interdiffusion à l'interface est accompagnée d'une interaction physicochimique et se traduit par la formation d'une couche d'interface de nature différente de celle du semiconducteur et du métal. Cette couche dont l'épaisseur croît avec la 
chaleur de réaction à l'interface peut induire des propriétés tout à fait différentes de celles du contact abrupt. En effet, elle introduit une interface nouvelle enterrée ; elle peut supporter, de plus, une différence de potentiel appréciable, assimilable à une modification d'affinité électronique.

Les propriétés du contact seront donc directement reliables à la réactivité de l'interface, les interfaces les plus ábruptes étant celles pour lesquelles l'interaction entre le métal et l'anion est la plus forte.

De nombreuses études ont été effectuées par Brillson dont certaines en déposant de très faibles couches métalliques en sandwich entre l'électrode métallique et le semiconducteur. Elles ont valorisé le modèle et mis en évidence des variations d'affinité interprétées par un transfert de charge entre deux plans atomiques, ou par la présence d'une couche plus épaisse dont la largeur a été évaluée par photoémission. Une question importante, celle de la croissance du métal, n'est pas prise en compte dans le modèle ; l'auteur suppose, en effet, une croissance sans îlot pour tous les métaux, ce qui ne correspond pas à la réalité.

4.2.5 Modèle de Freeouf-Woodall [57-59]. - Ce modèle intitulé «effective work function model» prend en compte la physicochimie de l'interface qui est supposée constitué de phases diverses caractérisées par des travaux de sortie propres. Dans cette hypothèse, l'interface est traitée en se basant sur le modèle de Schottky et la barrière est :

$$
\phi_{\mathrm{B}}=\phi_{\mathrm{eff}}-X
$$

$\phi_{\text {eff }}$ étant un travail effectif, obtenu par une moyenne pondérée des travaux de sortie des différentes phases en présence.

Dans le cas des siliciures, par exemple, le travail effectif s'exprime en fonction du travail de sortie du silicium $\phi_{\mathrm{Si}}$ et du métal par la relation $\left[\phi_{\mathrm{Si}}^{4} \phi_{\mathrm{m}}\right]^{1 / 5}$. Dans le cas des interfaces GaAs-Métal caractérisées par un excès d'As à l'interface, c'est le travail de sortie d'As élémentaire qui est pris en compte.

L'appréhension du modèle est relativement descriptive. Elle apporte cependant un éclairage original car elle prend en compte la non-uniformité de l'interface, ce qui correspond à de nombreux cas réels et, en particulier, au niveau des composants électroniques.

4.2.6 Autres modèles. - D'autres modèle ont été également proposés par Harrison [60], par Platero [61], par Hasagawa et Sawada [62]. Ces derniers considèrent que les états de surface ont, pour origine, un désordre résultant de variations aléatoires en longueur et en angle des liaisons.

Dans ces conditions, le spectre des états de surface serait continu et non discret comme le suppose le modèle des défauts.

\section{Discussion.}

$\mathrm{Si}$ le modèle des défauts est supporté par des résultats expérimentaux, il existe en fait une série importante de contre-exemples qui font que sa représentativité à fort recouvrement mais aussi à faible recouvrement est contestée [51, 63, 64, 65], en particulier parce que :

- sur GaAs à faible recouvrement, il est admis qu'on obtient le même ancrage pour les métaux réactifs du groupe III seulement;

- sur InP, la position de l'ancrage diffère suivant la réactivité du métal;

- pour un certain nombre de métaux, la barrière n'est pas établie lorsque le dépôt est inférieur à la monocouche. La croissance du film et l'apparition de ses propriétés métalliques se traduit par une possibilité d'écrantage beaucoup plus grande susceptible de modifier la répartition des charges entre le semiconducteur et les états de surface. L'ancrage n'est pas le même à faible recouvrement et en présence d'une couche épaisse ;

- les barrières dans les diodes sont parfois nettement modifiées par des réactions chimiques à l'interface [66] ;

- l'étude de nombreux composants montre que la majorité des états de surface, sinon la totalité, sont des états caractérisés par des constantes de temps très grandes [67], difficilement explicables si on prend en compte la position énergétique et la localisation des états induits.

Certaines de ces objections ont donné lieu à des travaux basés sur des modélisations de l'interface. A. Zur et al. [68] ont étudié l'ancrage à faible recouvrement et en présence d'une couche métallique épaisse, en se plaçant dans l'hypothèse d'une couche à l'interface perturbée par les défauts, d'épaisseur de quelques $\AA$ seulement. Ils ont montré qu'à faible recouvrement $10^{12}$ états par $\mathrm{cm}^{2}$ étaient nécessaires pour créer l'ancrage alors que, en présence d'une couche épaisse, il en fallait $10^{14}$ environ, la différence dans les positions de l'ancrage est expliquée par le fait que lorsque la densité des défauts est faible $\left(10^{11}\right.$ à $\left.10^{12} \mathrm{~cm}^{-2}\right)$, la charge dans ces états provient essentiellement du semiconducteur alors que, lorsque cette densité est élevée, cette charge est essentiellement compensée par la charge du métal, un dipôle se formant par la même occasion entre le métal et la couche.

Un modèle voisin présenté récemment par Spicer et al. [69] s'attache à l'analyse de l'interface en utilisant pour la représentation du métal un modèle atomique ou un modèle métallique et en plaçant des défauts sur un plan parallèle à l'interface et séparé d'elle par quelques $\AA$. Il contribue également à l'interprétation de certaines différences jusqu'alors incomprises dans l'ancrage de $E_{\mathrm{F}}$. 
D'autres modèles ont été également présentés ces dernières années.

J. M. Palau et al. [70] ont étudié un modèle supposant l'existence d'une couche mince localisée contre le métal et comportant des défauts accepteurs et donneurs de densité constante. Ils ont étudié l'évolution du diagramme énergétique, de la barrière et de l'ancrage et montré que l'hypothèse d'une telle couche rendait bien compte de la faible dépendance de la barrière avec le travail de sortie du métal même dans l'hypothèse de variations de ce dernier aussi importantes que $1 \mathrm{eV}$.

J. Y. Tang et al. [59] ont proposé une interprétation des résultats à partir des non-uniformités du travail de sortie, non-uniformités induisant des erreurs de mesures spécifiques à chaque technique, erreurs qu'il faut prendre en compte lors de la comparaison des résultats fournis par les différentes méthodes.

Il existe donc un faisceau d'explication qui s'ajoutent à celles d'ordre proprement physiques qui concernent la nature des défauts. L'étude des différentes possibilités associées aux états des liaisons pendantes, des lacunes, antisites... conduit souvent à une explication plausible.

Malgé ce, conclure à ce stade n'est pas, à l'heure actuelle, possible et ce, d'autant plus, que les modèles basés sur les états dans le gap induit par le métal (MIGS) apportent eux aussi des réponses raisonnables et qu'il faut prendre en compte même s'ils posent quelques difficultés pour répondre à un certain nombre de cas expérimentaux.

Comment, en effet, expliquer que l'ancrage soit le même sur des surfaces (110) (100) alors que la structure électronique de la surface est différente ? Comment expliquer que l'ancrage soit le même sur GaAs-Oxyde et GaAs-Métal ?
Comment justifier le rôle d'une couche d'oxygène adsorbée à l'interface métal-semiconducteur ?

Sans aller plus loin dans cette discussion, il est donc clair que, même si des modélisations récentes comme celles proposées par Lu (71) et basées sur la présence de MIGS, conduisent à des résultats théoriques en bon accord avec l'expérience, le point est loin d'être fait. Et aucun des deux précédents modèles ne peut objectivement faire l'unanimité.

Par ailleurs, il est certain que l'interaction chimique doit aussi être prise en compte et que des modèles comme ceux de Freeouf et Brillson ont également leur intérêt.

\section{Conclusion.}

Un point, à l'heure présente, sur le contact métalsemiconducteur conduit à conclure que la formation des défauts, l'interdiffusion, les réactions chimiques peuvent contribuer à la formation de l'interface. De ce fait, la mise au point de modèles s'avère complexe et ceux qui sont proposés, s'ils contribuent à une avancée dans la connaissance des mécanismes physiques, ne peuvent rendre compte de l'ensemble des faits observés. Le modèle unique, susceptible d'expliquer les différents cas de figure n'existe pas. Une étude synthétique s'avère donc indispensable. Mais elle ne peut reposer que sur des études à la fois plus fines et plus précises du contact, de sa formation et des propriétés des diodes dont l'analyse gagnerait à être plus poussée.

Ces études s'avèrent indispensables car, au plan technologique, le besoin d'un contact caractérisé par une barrière $\phi_{\mathrm{B}}$ donnée, ne peut être, à l'heure actuelle, satisfait. L' ingénierie » du contact n'est pas opérationnelle. Il faut encore développer les études amont sur les surfaces clivées mais aussi sur les surfaces « réelles » dont l'intérêt au plan de beaucoup de composants s'avère essentiel.

Bibliographie

[1] Physics and chemistry of III-V compounds semiconductors interfaces. Ed. Carl W. Wilmsen (Plenum Press) 1985.

[2] Metal-semiconductor barrier functions and their applications. Ed. B. L. Sharma (Plenum Press) 1984.

[3] SснотткY, W., Naturwissenshaften 26 (1938) 843.

[4] Mort, N. F., Note on the contact between a metal and an insulator or semiconductor. Proc. Cambridge Philos. Soc. 34 (1938) 568.

[5] Aven, M., Mead, C. A., Appl. Phys. Lett. 7 (1965) 8-10.

[6] Kurtin, S., McGill, T. C., Mead, C. A., Phys. Rev. Lett. 22 (1969) 1423-1436.

[7] Bardeen, J., Phys. Rev. 71 (1947) 717-727.
[8] Andrew, J. M., Phillips, J. C., Phys. Rev. Lett. 35 (1975) 56-59.

[9] BRIllson, L. J., Surf. Sci. Rep. 2 (1982).

[10] Freeouf, J. L., Rubloff, G. N., Ho, P. S., Kuan, T. S., J. Vac. Sci. Technol. 17 (1980) 916-925.

[11] Ottaviani, C., Tu, K. N., Mayer, J. W., Phys. Rev. Lett. 44 (1980) 284-287.

[12] Schluter, M., Phys. Rev. B 15 (1978) 5044.

[13] CoHen, M. L., J. Vac. Sci. Technol. 16 (1979) 1135.

[14] Cowley, A. M., Sze, S. M., J. Appl. Phys. 36 (1965) 3212.

[15] Nicollian, E. W., Schwartz, B., Coleman, D. J., RYDER, R. M., BREWS, J. R. J. Vac. Sci. Technol. 13 (1976) 1047. 
[16] Mayer, R. J., Duke, C. B., Platon, A., Kahn, A., So, E., Yeh, J. L., Mark, P., Phys. Rev. B 19 (1979) 5194-5202.

[17] Bertoni, C. M., Bisi, O., Calandra, C., Mangi, F., Physics of Semiconductors, Int. Phys. Conf. Ser. 43 (1978) 191.

[18] Palau, J. M., Testemale, E., Lassabatere, L., $J$. Vac. Sci. Technol. 19 (1981) 192-200.

[19] Huijser, A., VAN LAAR, J., Surf. Sci. 52 (1975) 202210.

[20] Montgomery, V., Srivastava, G. P., Sing, I., Williams, R. H., J. Phys. C. 16 (1983) 36273640.

[21] Guichard, G. M., Thèse d'Etat, Paris, 1978.

[22] Gatos, H. C., Lagowski, J., J. Vac. Sci. Technol. 10 (1973) 130.

[23] Ismail, A., Palau, J. M., Lassabatere, L., J. Physique 45 (1984) 1717-1723.

[24] MORRISON, S. Ray, The chemical physics of surfaces (Plenum Press, New York-London) 1978.

[25] BAuer, E., Z. Kristallogr. 110 (1958) 372.

Bauer, E., Poppa, H., Thin Solid Films 12 (1972) 167.

[26] Derrien, J., Arnaud D'Avitaya, F., Solid State Comm. 20 (1976) 557-560. Surf. Sci. 65 (1977) 668-686.

[27] Massies, J., LinH, N. T., J. Crystal Growth 56 (1982) 25.

[28] Houzay, F., Bensoussan, M., Barthe, F., Surf. Sci. 168 (1986) 347-355.

[29] Mednick, K., Kleiman, L., Phys. Rev. B 22 (1980) 5768.

[30] Caruthers, E. B., Kleiman, L., Alldredge, G. P., Phys. Rev. B 9 (1974) 3330.

[31] Ismail, A., Thèse d'Etat, Montpellier (1984).

[32] Bolmont, D., Chen, P., Mercier, V., Sebenne, C., Proceeding 16th International Conference on the Physics of Semiconductors, 1982.

Bolmont, Thèse d'Etat, Paris 1982.

[33] Spicer, W. E., Kendelewicz, T., Newman, N., Chin, K. K., Lindau, I., Surf. Sci. 168 (1986) 240.

[34] Williams, R. H., Physics and Chemistry of III-V compound semiconductor interfaces (Plenum Press) 1985 , p. 1-72.

[35] Rhoderick, E. H., Metal-semiconductor contacts (Clarindon Press, Oxford) 1978.

[36] TyAgI, M. S., dans la référence 2.

[37] Robinson, Gary Y., dans la référence 1.

[38] McKinley, A., Hugues, G. J., Williams, R. H., J. Phys. C 10 (1977) 4545-4555.

[39] Tersoff, J., Phys. Rev. Lett. 52 (1984) 465.

[40] Spicer, W. E., Lindau, I., Skeath, P., Su, C. Y., Chye, P., Phys. Rev. Lett. 44 (1980) 420-423.

[41] Spicer, W. E., Lindau, I., SKeath, P., Su, C. Y., J. Vac. Sci. Technol. 17 (1980) 1019-1027.

[42] Allen, R. E., Dow, J. D., Phys. Rev. B 25 (1982) 1423.
[43] Allen, R. E., Humphreys, T. J., Dow, J. D., JANKEY, O. F., J. Vac. Sci. Technol. B 2 (1984) 449-452.

[44] Allen, R. E., Jankey, O. F., Dow, J. D., Surf. Sci. 168 (1986) 376-385.

[45] Heine, V., Phys. Rev. A 138 (1965) 1689-1696.

[46] Pellegrini, B., Solid State Electron. 17 (1974) 213237.

[47] Inkson, J. C., J. Phys. C. 6 (1973) 1350-1362.

[48] Louie, S. G., Cohen, M. L., Phys. Rev. B 13 (1976) 2461.

[49] Louie, S. G., Chelikowski, J. R., Cohen, M. L., Phys. Rev. B 15 (1976) 2145.

[50] Louie, S. G., Chelikowski, J. R., Cohen, M. L., Phys. Rev. B 15 (1977) 2154.

[51] Tersoff, J., Surf. Sci. 168 (1986) 275.

[52] Tersoff, J., J. Vac. Sci. Technol. B 3 (1985) 1157.

[53] Lefebvre, I., Lannoo, M., Priester, C., Allan, G., The role of dangling bonds at Schottky barriers and semiconductor heterojunctions. To be published.

[54] LANNOO, M. et al. Journées Microélectroniques IIIV, janvier 1987. A publier dans la Revue de Physique Appliquée.

[55] Brillson, L. J., Brucker, C., Stoffel, N. G., Katnani, A. D., Margaritondo, G., Phys. Rev. Lett. 46 (1981) 838.

[56] Brillson, L. J., Brucker, C. F., Katnani, A. D., Stoffel, N. G., DANiels, R., MARGariTONDO, G., PCSI, Asilomar, Californie, 1982.

[57] Woodall, J. M., Freeouf, J. L., J. Vac. Sci. Technol. 19 (1981) 794-798.

[58] Freeouf, J. L., Woodall, J. M. Appl. Phys. Lett. 39 (1981) 727-729.

[59] Tang, J. Y., Freeouf, J. L., J. Vac. Sci. Technol. B 2 (1984) 459.

[60] Harrison, W. A., Phys. Rev. Lett. 37 (1970) 312.

[61] Platero, G., Verges, J. A., Flores, F., Surf. Sci. 168 (1986) 100-104.

[62] Hasegawa, H., Sawada, T., Thin Solid Film 103 (1983) $119-140$.

[63] Schaffer, F., AbStreiter, G., J. Vac. Sci. Technol. B 3 (1985) 1184-1189.

[64] BACHRACH, R. Z., Metal semiconductor Schottky barrier junction and their applications. B. L. Sharma Ed. (Plenum Press) 1984.

[65] Lile, D., J. Vac. Sci. Technol. B 2 (1984) 496-503.

[66] Waldrop, J. R., J. Vac. Sci. Technol. B 3 (1985) 1197-1201.

[67] Lile, D. L., Taylor, M. J., J. Appl. Phys. 54 (1983) 260.

[68] Zur, A., McGill, T. C., SMith, D. L., Phys. Rev. B 28 (1983) 2060.

[69] Spicer, W. E., Pan, S., Mo, D., Newman, N., Mahowald, P., Kendelewicz, T., Eglash, S., J. Vac. Sci. Technol. B 2 (1984) 476-480.

[70] Palau, J. M., Ismail, A., Lassabatere, L., Solid State Electron. (1985) 499-508.

[71] Lu, G. N., Thèse, Orsay, décembre 1986. 